\title{
Influence of azelaic and mandelic acid peels on sebum secretion in ageing women
}

\author{
Aneta Wójcik, Marlena Kubiak, Helena Rotsztejn \\ Department of Cosmetology, Medical University of Lodz, Poland \\ Head: Prof. Ryszard Glinka MD, PhD
}

Postep Derm Alergol 2013; XXX, 3: 140-145

DOI: 10.5114/pdia.2013.35614

\begin{abstract}
Introduction: Azelaic acid and mandelic acid are superficial peels commonly applied in people of various age groups. As they are mild and do not cause any side effects, they are also often used in elderly people.

Aim: To compare the influence of azelaic and mandelic acid peels on facial sebum secretion in mature women aged 49-71 years.

Material and methods: The level of secreted sebum was measured in 28 women. Eleven women were treated with azelaic acid peel and 17 with mandelic acid peel. Each of the peels was applied five times with 2-week intervals. The measurements were made on the cheeks and chin with the use of Sebumeter SM 15 (Courage \& Khazaka, Germany). The last measurement, i.e. the sixth one, was made 2 weeks after the treatment.

Results: We observed a significant increase in sebum secretion in the U-zone after the application of $20 \%$ azelaic peel and $40 \%$ mandelic peel. Neither peel significantly affected sebum secretion in the T-zone.

Conclusions: Peels with 20\% azelaic acid and 40\% mandelic acid might be considered treatments which contribute to an increase in sebum secretion in ageing women.
\end{abstract}

Key words: azelaic peel, mandelic peel, ageing, sebumeter.

\section{Introduction}

With time skin loses its elasticity; it becomes thin and dry. Wrinkles and discoloration are clearly visible. This results from the fact that reaction sites are not activated any more as the level of sex hormones, especially estrogens, is low. The chronological ageing is accompanied by photoaging, which additionally impairs the skin functions [1-3]. Chemical peels are applied to assist in the elimination of the effects of skin ageing. They make cells proliferate and become metabolically more active as well as influence the skin lipids. The last factor keeps the skin in good condition [4]. Acid peels applied to the skin activate specific mechanisms, both chemical - amide bond hydrolysis and biochemical - inflammatory reaction. The chemical mechanism involves removing the existing epidermal cell structures and replacing them with the new ones, after an earlier release of cytokines and mediators by keratinocytes. Interleukin-1 $\alpha$ induces the release of interleukin-6, which stimulates fibroblasts to increase the production of matrix metalloproteinases (MMPs). They are involved in the connective tissue remodelling process during which hyalur- onic acid and new collagen fibres production grows. It stimulates the natural regeneration processes in the skin, leading to the improvement of its physiological properties [1-4].

There are not many studies on effects of different peels on sebum secretion in ageing women.

\section{Aim}

The aim of the study was to evaluate the influence of azelaic or mandelic acid superficial peels on the skin of perimenopausal women and their effects on minimising the discomfort connected with dryness, desquamation and sometimes burning of the skin.

\section{Material and methods \\ Patient population}

The study group consisted of 28 women. Eleven women were treated with $20 \%$ azelaic acid peel and 17 with $40 \%$ mandelic acid peel. The patients were aged 49-71 (mean age \pm SD: $60.4 \pm 6.4$ ). The skin types of our patients

Address for correspondence: Prof. Helena Rotsztejn, Department of Cosmetology, Medical University of Lodz, 1 Muszynskiego St, 90-151 Lodz, Poland, phone: +48 604180 044, fax: +48 4267791 14, e-mail: rotsztejn@onet.eu Received: 18.10.2012, accepted: 10.02.2013. 
Table 1. Age of the patients according to the peel

\begin{tabular}{lccccccccc}
\hline Peel & $N$ & Min. & Max. & Median & Mean & SD & SEM & CV\% & Value of $p$ \\
\hline Total $N$ & 28 & 49.0 & 71.0 & 61.5 & 60.4 & 6.4 & 1.2 & 10.6 \\
\hline Azelaic (A) & 11 & 49.0 & 66.0 & 58.0 & 58.5 & 5.6 & 1.7 & 9.5 \\
\hline Mandelaic (M) & 17 & 49.0 & 71.0 & 62.0 & 61.5 & 6.8 & 1.6 & 11.0 & $>0.05$ \\
\hline
\end{tabular}

$N$-number of patients

were FitzPatrick types II and III, Glogau photoaging scale types III and IV.

Each of the subjects was informed about the method of performing the study and the way of home skin care. Patients were instructed to apply a moisturizing cream twice a day for a week, to avoid sun exposure, and to use sunscreens daily. None of the patients changed skin care cosmetics in the period in which the study was conducted and they had not taken antibiotics or retinoids or hormonal therapy for 6 months before the treatment. Patients with a history of hypertrophic scarring, keloids, active or recurrent herpes and oral isotretinoin therapy within the previous 6 months were excluded from the study (Table 1).

\section{Procedure}

The measurements were made on the skin of the face - the forehead, left and right cheek and chin with the use of a diagnostic device - Sebumeter SM 15 (Courage \& Khazaka, Germany). To evaluate the level of sebum we used a special cassette with a parchment tape. It was pressed to selected areas of the face with a force of $4 \mathrm{~N}$ for a period of $30 \mathrm{~s}$ (according to the manufacturer's instructions). Next, the tape soaked with sebum was photometrically analyzed in the probe of MP 15 device [5-7]. The area of the skin was divided into two zones - one where the sebum was secreted more intensively (T-zone: the forehead, chin) and the other where the secretion was less active ( $U$-zone: the left and right cheek) [8-10]. The data were gathered and stored in the data base. All the measurements were made in the same room at temp. $20^{\circ} \mathrm{C}$ and humidity $40-60 \%$. The study consisted of 5 treatment sessions performed every 2 weeks. The sebum level was measured during the first visit before the application of the peel. The same procedures were repeated during each visit. The last measurement was made 2 weeks after completing the study. In total the patients' sebum level was measured six times. Before the application of the selected kind of peel, the facial skin was cleansed with water with mild soap, dried and left without applying any cream. Next, $5 \mathrm{~h}$ later, we measured the level of sebum on the forehead, chin, left and right cheek. Before the treatment the skin was degreased with 70\% isopropyl alcohol and thoroughly dried. A surgical cap was used to pull back the hair and cover the ears, while the eye, nostrils and mouth areas were protected with Vaseline. During the procedure, azelaic or mandelic acids were applied on the whole face for 5 min to $10 \mathrm{~min}$. The treatment time depended on the intensity of the clinical response, skin type and the Glogau-Fitzpatrick index. The peel was neutralized with $10 \%$ sodium bicarbonate in water solution. Next, the face was washed twice, dried and moisturizing cream was applied. The patients were sent home and asked to apply moisturizing cream twice a day and avoid exposing to the sun. All patients were strongly encouraged to use a sunscreen.

\section{Statistical analysis}

Statistical analysis was conducted with Statistica 5. For the data expressed in the interval scale, we calculated minimum and maximum, mean, median, standard deviation, standard error of the mean as well as 95\% coefficient intervals for the means. Normal distribution was checked with Shapiro-Wilk test.

TStudent test for independent samples was used to make comparisons between independent groups in which parameters did not significantly differ from the normal distribution (with an adopted significance level $\alpha=0.05$ ). If the hypothesis of normal distribution or parameters in the rank scale was rejected, we used $U$ Mann-Whitney test.

The comparison of dependent samples (sebum concentration at the time point) was made with a single-factor variance analysis. If it turned out to be unreliable, the authors replaced it with non-parametric equivalent, i.e. Friedman's test. Next, to evaluate differences between selected time points, $t$ Student test for independent samples was used. If it appeared to be unsuccessful, Wilcoxon test was applied.

The diagrams present mean values with $95 \%$ coefficient intervals and significant differences between the values of the base level and the remaining levels at subsequent time points for azelaic and mandelic peels.

\section{Results}

Eleven women were treated with azelaic acid peel and 17 with mandelic acid peel. All 28 patients completed the study. The diagrams show a detailed activity of the two peels at particular time points as a mean value with 95\% coefficient intervals.

\section{Azelaic peel}

Before the application of azelaic peel, the mean level of sebum secretion on the left cheek for the base measurement was $31.45 \pm 33.27 \mu \mathrm{g} / \mathrm{cm}^{2}$. A statistically significant increase, up to $51.82 \pm 30.89 \mu \mathrm{g} / \mathrm{cm}^{2}$, was observed after the fifth procedure $(p<0.05)$ and it was equal to 


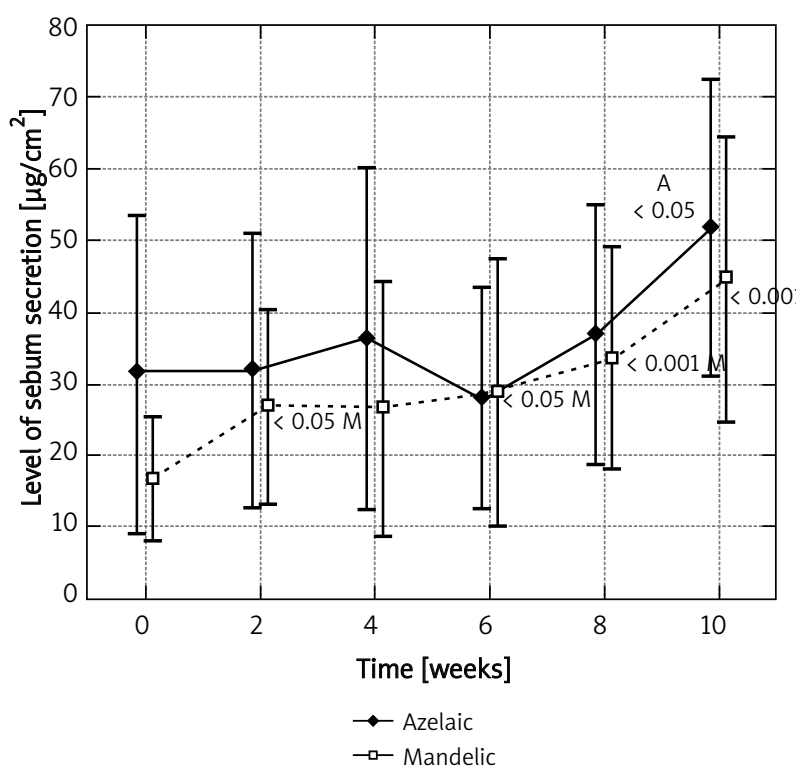

Fig. 1. Levels of sebum secretion measured on the left cheek at time points for azelaic and mandelic peels

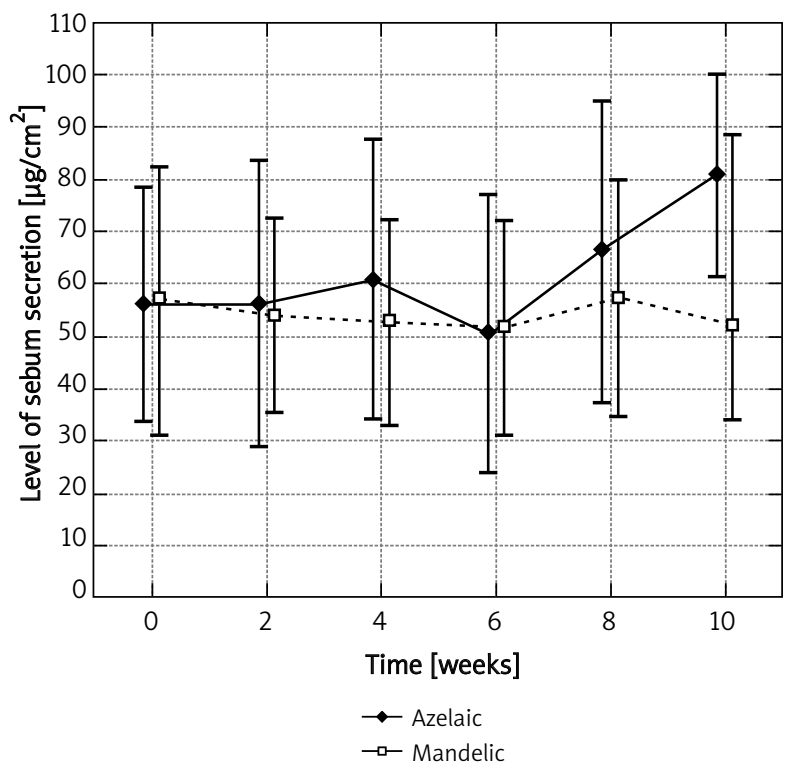

Fig. 3. Levels of sebum secretion measured on the chin at time points for azelaic and mandelic peels

$164.7 \%$ of the base level (Figure 1). A similar observation was noted on the right cheek, where azelaic peel was significantly effective as early as after the fourth procedure. Then, the base level was $48.27 \pm 59.89 \mu \mathrm{g} / \mathrm{cm}^{2}$ and the final level $-75.82 \pm 38.93 \mu \mathrm{g} / \mathrm{cm}^{2}$, which represented $57.1 \%$ of the base value (Figure 2). Azelaic acid contributed to a $44.5 \%$ increase in the secretion of sebum on the chin and a $18.6 \%$ secretion on the forehead, which in both cases was not statistically significant (Figures 3 and 4). The variance

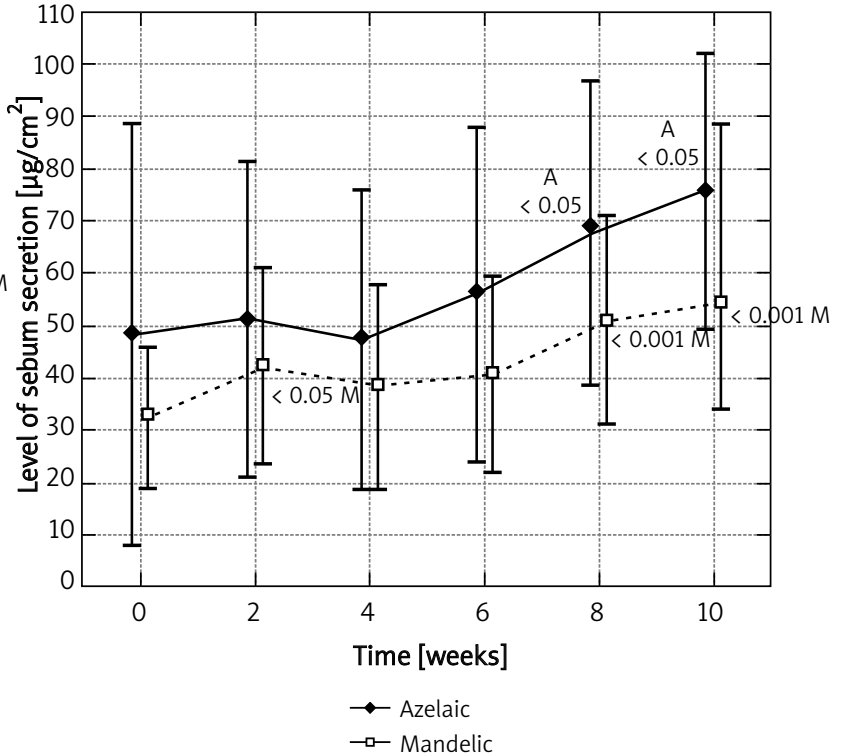

Fig. 2. Levels of sebum secretion measured on the right cheek at time points for azelaic and mandelic peels

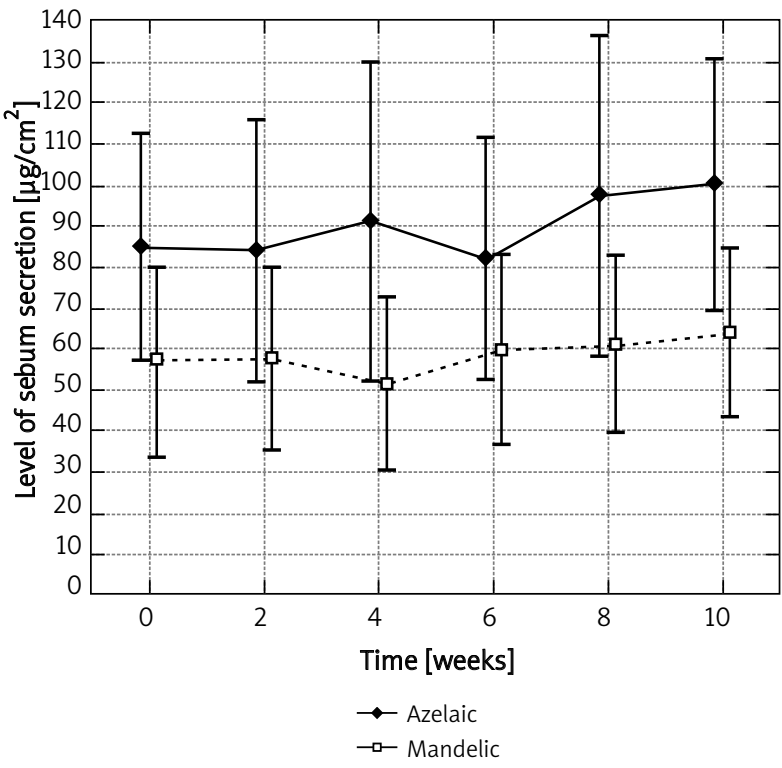

Fig. 4. Levels of sebum secretion measured on the forehead measured at time points for azelaic and mandelic peels

analysis confirmed a significant difference between level measurements at particular time points for azelaic peel on the right cheek $p<0.05$ and on the left cheek $-p<0.05$. No statistical difference was observed for azelaic peel applied on the chin or the forehead $(p>0.05)$.

Having applied four treatment sessions with azelaic acid every 2 weeks, we noted a statistically significant increase in sebum secretion in the $U$-zone. The mean value grew from $39.86 \pm 48.06 \mu \mathrm{g} / \mathrm{cm}^{2}$ to $63.82 \pm 36.42 \mu \mathrm{g} / \mathrm{cm}^{2}$, which 


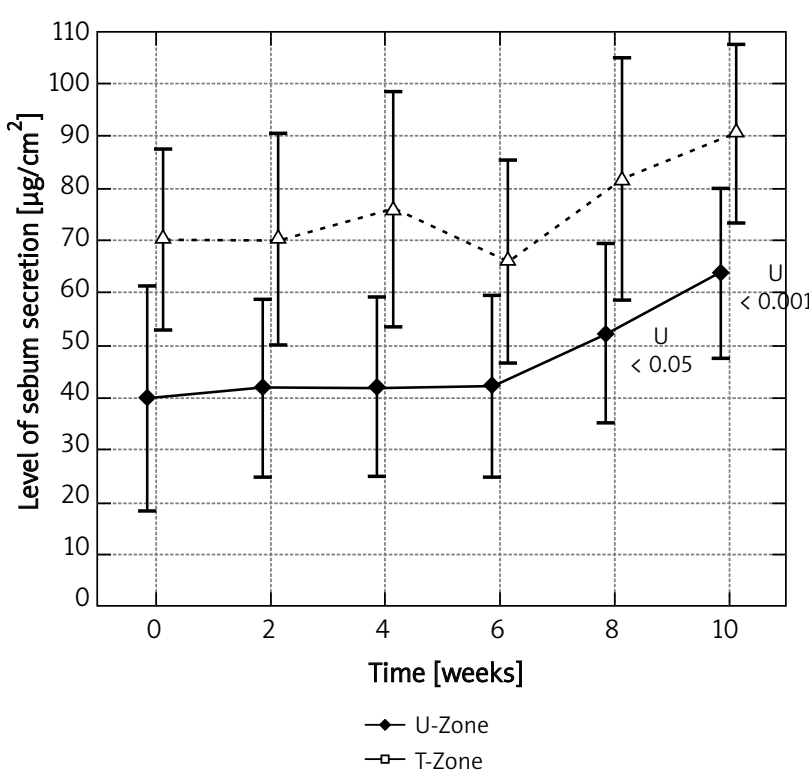

Fig. 5. Levels of sebum secretion for $U$ and $T$ zones measured at time points for azelaic peel

signified an increase by $60.1 \%$ in comparison with the initial measurement. With regard to the T-zone, an increase was observed after five treatment sessions, so after the whole treatment period (10 weeks) the final level grew by $28.9 \%$ in comparison to the initial level. Figure 5 presents statistically significant values

The variance analysis confirmed a statistically significant difference between the levels measured at the studied time points for azelaic peel in the U-zone $(p<0.001)$. In the T-zone no statistically significant differences were observed.

\section{Mandelic acid}

Mandelic acid showed an increase in sebum secretion on the left cheek by $168.1 \%$. The mean value of sebum secretion before the first treatment session was $16.59 \pm 16.63$ $\mu \mathrm{g} / \mathrm{cm}^{2}$ and after 10 weeks - it was $44.47 \pm 38.70 \mu \mathrm{g} / \mathrm{cm}^{2}$. The results confirmed a significant increase in sebum secretion already after the first session $(p<0.05)$, where in the final measurement, the peel appeared to be significantly effective in comparison with the initial measurement $(p<0.001)$.

Two weeks following the first session a statistically significant increase in sebum secretion was observed on the right cheek $(p<0.05)$. An increase in the mean value of sebum secretion was noted 2 weeks after the completion of the treatment. It grew from $32.65 \pm 2652 \mu \mathrm{g} / \mathrm{cm}^{2}$ to $54.41 \pm 39.29$ $\mu \mathrm{g} / \mathrm{cm}^{2}$, which means an increase by $66.7 \%$, with the adopted significance $(p<0.001)$. The application of mandelic peels on the chin did not contribute to changes in the level of the secreted sebum. An increase on the forehead by $12.6 \%$ appeared to be statistically insignificant $(p>0.05)$. The variance analysis showed a significant difference between the

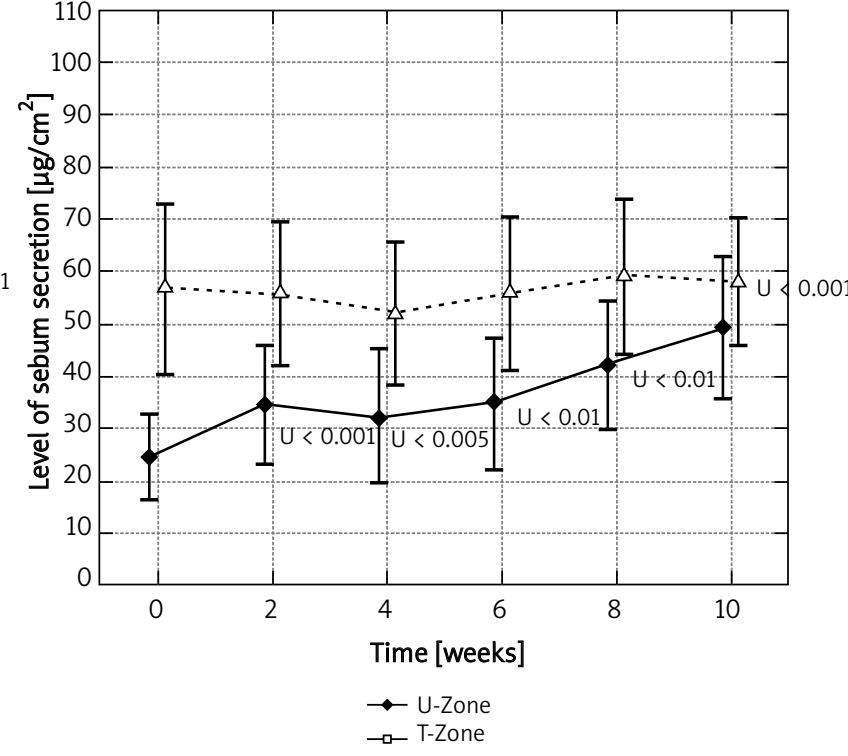

Fig. 6. Levels of sebum secretion for $U$ and $T$ zones measured at time points for mandelic peel

levels measured at the studied time points for mandelic peel on the right and left cheek $(p<0.001)$.

Mandelic peel did not contribute much to sebum secretion in the T-zone (by $2 \% ; p>0.05 \mathrm{NS}$ ). In the $\mathrm{U}$-zone significant differences were observed already after 2 weeks. They remained until the end of the treatment $(p<0.001)$ (Figure 6).

The variance analysis confirmed a statistically significant difference between the levels measured at the studied time points for mandelic peel in the $\mathrm{U}$-zone $(p<0.001)$. In the T-zone no statistically significant differences were observed.

\section{Discussion}

Azelaic acid is an important therapeutic agent applied not only in common acne, but also in skin discolorations, mainly in melasma and acne rosacea - the disorders common for ageing women [11-15]. By inhibiting the activity of neutrophils, it inhibits the production of free radicals and serves as their scavenger. Thus, it has anti-ageing properties [11]. By blocking oxidoreductive mitochondrial enzymes and inhibiting the synthesis of DNA and bacteria cellular proteins, azelaic acid acts bacteriostatically on Propionibacterium acnes, Staphylococcus aureus and Staphylococcus epidermidis $[13,14,16]$. By inhibiting $5 \alpha$ reductase - the enzyme catalyzing the reaction of changing testosterone to its active form - dihydrotestosterone it decreases lipogenesis. Thus, it has comedolytic properties [13]. However, the influence of azelaic acid has not been fully determined [17]. Physical and chemical properties of mandelic acid, slow penetration and therefore, a more effective control of the process of penetration dur- 
ing a procedure and the fact that it is well tolerated by patients with sensitive skin, contributed to the popularity of the acid. It is commonly used in dermatology and aesthetic medicine, in treating various kinds of acne, tanning discolorations as well as in anti-wrinkle procedures. An aromatic ring in the structure of mandelic acid makes it a compound of lipophilic properties. In acidic environment it demonstrates bacteriostatic and bactericidal properties, especially for the following bacteria strains: Staphylococcus aureus, Bacillus proteus, Escherichia coli and Aerobacter aerogenes [18].

There are not many studies on the application of superficial peels such as: $\alpha$-hydroxyacid (30\% glycolic acid), Jassner solution, $\beta$-hydroxyacid (30\% salicylic acid), 50\% pyruvic acid and their effect on sebum secretion. The influence of superficial peels - on sebum secretion, especially in ageing women has not been fully elucidated.

Lee et al. studied the influence of superficial peels $30 \%$ glycolic acid and Jassner solution on sebum secretion in patients with acne. The results confirmed that both peels did not considerably contribute to a decrease in sebum secretion in the studied group [19]. Lee et al. studied an influence of $30 \%$ salicylic peel on the improvement of therapeutic effects in common acne. The results proved that salicylic acid brought positive effects in the treatment of disorders in the mentioned group. The researchers did not analyze any relationships with sebum secretion [20].

In addition, Ahn and Kim showed salicylic acid to have a whitening effect on the skin [21]. This effect was seen in the study of Garg and Sinha, too, with patients reporting diffuse lightening of their facial complexion. Mandelic acid has also been shown to be effective in clearing hyperpigmentation in patients who were resistant to other conventional modalities [22].

Kessler et al. conducted a randomized double blind trial. It aimed at comparing the efficacy of chemical peels, $\alpha$ - and $\beta$-hydroxyacids, in treating mild and moderate common acne. Both peels, $\alpha$ - and $\beta$-hydroxyacids, appeared to be efficient agents in treating acne. However, the authors did not analyze any relationships with sebum secretion [23].

Based on instrumental evaluation, using corneometer, cutometer, mexameter and visioscan, Berardesca et al. studied the effects of 50\% pyruvic acid after four treatment sessions performed once in 2 weeks. The results showed a significant reduction in the degree of pigmentation in patients with melasma, a significant increase in skin elasticity and a reduction of wrinkles in all the patients [24].

Garg et al. compared the therapeutic efficacy and tolerability of $35 \%$ glycolic acid and $20 \%$ salicylic- $10 \%$ mandelic acid peels in active acne and post acne scarring and hyperpigmentation. In this study, salicylic-mandelic acid combination peels (SMPs) were seen to be significantly better than glycolic acid peels in the treatment of non-inflammatory lesions and hypersecretion of sebum. This is because of the unique lipophilic and anti-inflammatory properties of both acids.
In other studies, the authors evaluated the relationship between sebostatic effectiveness, tolerance and activity of three topical drugs: azelaic acid, benzoyl peroxide, adapalene in treating mild and moderate acne. All the three drugs appeared to bring good therapeutic effects and caused hardly any side effects. Their activity, however, does not seem to be correlated with sebostatic activity [25].

Our study confirmed that in the group of ageing women, azelaic and mandelic peels contribute to an increase in sebum secretion in the $U$-zone. In the T-zone no significant differences were observed. The level of sebum secretion depends on the area of the face. It is topographically dependent. It is commonly known that sebum secretion is higher in the T-zone rather than in the $U$-zone $[9,10]$. In the studied group, the increase appeared on some face parts - the left and right cheek, where the initial level of sebum secretion was lower than the level of sebum secretion on the forehead and the chin. Both peels, with azelaic and mandelic acids, significantly contributed to sebum secretion on both cheeks. With regard to mandelic acid, there was an increase already after 2 weeks following the first treatment session, both on the left and right cheek. After the application of azelaic acid, an increase was observed after 8 weeks, i.e. after the fourth treatment session (the right cheek) and after 10 weeks, i.e. after the fifth treatment session (the left cheek). There were no significant differences between the two chemical peels with regard to sebum secretion in the T-zone, from the initial level to the end of the study.

Chemical peels with azelaic and mandelic acids were well tolerated by the whole studied group. No immediate or long-term side effects were observed. After the application of mandelic acid, slight exfoliation was observed. It remained for seven up to 10 days and was eliminated by using moisturizing creams.

Although both of the agents led to a subtle increase in the sebum secretion in ageing women, the difference between the two did not appear to be clinically significant. Because both of the peeling agents are superficial peels, they only serve to resurface the upper layers of the epidermis. Through an indirect, yet unknown, mechanism they both stimulate the dermal fibroblasts to deposit more collagen, elastin and GAGs in the papillary dermis and also stimulate production of sebum in ageing women.

\section{Conclusions}

We can say that peels with azelaic and mandelic acids may be considered an effective, safe and well-tolerated method in ageing women. They can reduce effects of ageing processes, especially in dryness by regulating sebum secretion in the areas where it is considerably reduced.

\section{References}

1. Wójcik A, Rotsztejn $\mathrm{H}$. The influence of hormones on skin lipids in women during menopause. Prz Menopauzalny 2012; 11: 41-4. 
2. Wines N, Willsteed E. Menopause and the skin. Austral J Dermatol 2001; 42: 149-60.

3. Callens A, Vaillant L, Lecomte P, et al. Does hormonal skin aging exist? A study of influence of different hormone therapy regimens on the skin of postmenopausal womenusing non-invasive measurement techniques. Dermatology 1996; 193: 289-91.

4. Grimes PE, Green BA, Wildnauer RH, et al. The use of polyhydroxy acids (PHAs) in photoaged skin. Cutis 2004; 73 (Suppl 2): 3-13.

5. Wójcik A, Budzisz E, Rotsztejn H. Skin surface lipids and their measurement. Postep Derm Alergol 2011; 28: 498-505.

6. Thune P, Gustavsen T. Comparison of two photoelectric techniques for quantitative measurements of skin surface lipids. Acta Derm Venereol 1987; 134: 30-2.

7. Dikstein S, Zlotogorski A, Avriel E, et al. Comparison of the sebumeter and the lipometer. Bioeng Skin 1987; 3: 197.

8. Youn SW, Na JI, Choi SY, et al. Regional and seasonal variations in facial sebum secretions: a proposal for the definition of combination skin type. Skin Res Technol 2005; 11: 189-95.

9. Park SG, Kim YD, Kim JJ, Kang SH. Two possible classifications of facial skin type by two parameters in Korean women: sebum excretion rate (SER) and skin surface relief (SSR). Skin Res Technol 1999; 5: 189-94.

10. Pagnoni A, Kligman AM, el Gammal S, Stoudemayer T. Determination of density of follicles on various regions of the face by cyanoacrylate biopsy: correlation with sebum output. $\mathrm{Br}$ J Dermatol 1994; 131: 862-5.

11. Gibson JR. Rationale for the development of new topical treatments for acne vulgaris. Cutis 1996; 57 (Suppl 1): 13-9.

12. Breathnach AS. Melanin hyperpigmentation of skin: melasma, topical treatment with azelaic acid, and other therapies. Cutis 1996; 57 (Suppl. 1): 36-45.

13. Holland KT, Bojar RA. The effect of azelaic acid on cutaneous bacteria. J Dermatol Treat 1989; 1: 17-9.

14. Bladon PT, Burke BM, Cunliffe WJ, et al. Topical azelaic acid and the treatment of acne: a clinical and laboratory comparison with oral tetracycline. Br J Dermatol 1986; 114: 493-9.

15. Carmichael AS, Marks R, Graupe KA, et al. Topical azelaic acid in treatment of rosacea. J Dermatol Treat 1993; 4 (Suppl. 1): 19-24.

16. Holland KT, Bojar RA. Antimicrobial effects of azelaic acid. J Dermatol Treat 1993; Suppl 1: 8-11.

17. Youn SW. The role of facial sebum secretion in acne pathogenesis: facts and controversies. Clin Dermatol 2010; 28: 8-11.

18. Taylor MB. Summary of mandelic acid for the improvement of skin conditions. Cosmet Dermatol 1999; 6: 26-8.

19. Lee SM, Huh CH, Park KC, Youn S.W. Effects of repetitive superficial peels on facial sebum secretion in acne patients. J Eur Acad Dermatol Venereol 2006; 20: 964-8.

20. Ho-Sup L, Il-Hwan K. Salicylic acid peels for the treatment of acne vulgaris in Asian patients. Dermatol Surg 2003; 29 : 1196-9.

21. Ahn HH, Kim KH. Whitening effect of salicylic acid peels in Asian patients. Dermatol Surg 2006; 32: 372-5.

22. Garg VK, Sinha S. Glycolic acid peels versus salicylic-mandelic acid peels in active acne vulgaris and post-acne scarring and hyperpigmentation. Dermatol Surg 2009; 35: 59-65.

23. Kessler E, Flanagan K, Chia Ch, et al. Comparison of alpha and beta hydroxyl acid chemical peels in the treatment of mild to moderately severe facial acne vulgaris. Dermatol Surg 2008; 34: 45-51.
24. Berardesca E, Cameli N, Primavera G, Carrera M. Clinical and Instrumental evaluation of skin improvement after treatment with a new 50\% puruvic acid peel. Dermatol Surg 2006; 32: 526-31

25. Stinco G, Bragadin G, Trotter D, Pillon B, Patrone P. Relationship between sebostatic activity, toler ability and efficacy of three topical drugs to treat mild tomoderate acne. J Eur Acad Dermatol Venereol 2007; 21: 320-5. 